\title{
System Identification of Rhythmic Hybrid Dynamical Systems via Discrete Time Harmonic Transfer Functions
}

\author{
M. Mert Ankarali and Noah J. Cowan
}

\begin{abstract}
Few tools exist for identifying the dynamics of rhythmic systems from input-output data. This paper investigates the system identification of stable, rhythmic hybrid dynamical systems, i.e. systems possessing a stable limit cycle but that can be perturbed away from the limit cycle by a set of external inputs, and measured at a set of system outputs. By choosing a set of Poincaré sections, we show that such a system can be (locally) approximated as a linear discrete-time periodic system. To perform input-output system identification, we transform the system into the frequency domain using discretetime harmonic transfer functions. Using this formulation, we present a set of stimuli and analysis techniques to recover the components of the HTFs nonparametrically. We demonstrate the framework using a hybrid spring-mass hopper. Finally, we fit a parametric approximation to the fundamental harmonic transfer function and show that the poles coincide with the eigenvalues of the Poincaré return map.
\end{abstract}

\section{INTRODUCTION}

In this paper, we propose a framework for system identification of rhythmic hybrid dynamical systems around their limit cycles. Rhythmic dynamic behaviors can be observed in a wide variety of biological and robotics systems, such as terrestrial locomotion [3,17] and juggling [2,4]. Such behaviors often include hybrid characteristics in that they exhibit both smooth flows punctuated by discrete jumps and are often modeled as hybrid dynamical systems [10].

Powerful analytical and numerical tools have been developed in order to control and analyze such hybrid dynamical systems and behaviors $[1,7,10]$. However, these tools are limited to the case when we have a full (and preferably simple) mathematical model-typically derived from first principles - that can accurately describe the system dynamics, but such modeling requires many creative decisions about what to neglect. More critically, it is impossible to derive equations, from first principles, that capture the influence of the nervous system on these hybrid dynamics $[8,19]$.

In the context of non-rhythmic dynamical systems near equilibria, system identification is a mature field [18]. System ID provides a very powerful complement to modeling systems using first principles.

By contrast, system ID for rhythmic and/or hybrid systems remains radically limited. Several researchers have addressed system ID and analysis of rhythmic systems by studying the steady state behavior and synthesizing these results using dynamical systems language [13]. However, this type of

M. Mert Ankarali is with the Dept. of Mechanical Eng.,Johns Hopkins University, 21218 Baltimore, MD, USA mertankarali@ jhu. edu

Noah J. Cowan is with the Dept. of Mechanical Eng.,Johns Hopkins University, 21218 Baltimore, MD, USA approach is extremely limited for answering the question of such as how these systems recover from perturbations.

There are also recent studies that attempt to estimate and quantify the dynamics around the limit cycles of such systems from data $[2,15]$. The most popular mathematical framework used in such analysis is based on Poincaré return maps which reduce the rhythmic dynamical system to a lower dimensional discrete-time system that describes the behavior in terms of its cycle-to-cycle transitions. Linearization of this reduced system yields a discrete time LTI system. The power of Poincaré theory is that it connects rhythmic dynamical systems to LTI systems theory, affording rich and powerful tools for both analysis and identification. However, the obvious limitation of this approach is that only one measurement per cycle is used. The approach lumps all effects within a cycle together, making it difficult to relate system-level dynamics with the roles of individual component-level details. Relatedly, there is a severe loss in temporal resolution. In addition, the approach presumes access to all state variables, an assumption not realistic when dealing with neural control systems.

In order to resolve some of these issues, Revzen et al. [15] introduced an identification framework inspired by Floquet theory and which utilizes multiple sections within a cycle and identifies mappings between them. However, this approach is "input free" and the identification is performed only based on the output measurements (and thus doesn't address the problem of hidden states). Typically, for the identification of dynamical systems input-output methods are more powerful and accurate compared to output only methods.

Kiemel et al. [12] proposed a new formulation that addresses the input-output identification of rhythmic systems around their limit cycles in the frequency domain. Specifically they approximate the dynamics near the limit cycle as a linear time-periodic (LTP) system. In this framework, they non-parametrically estimate LTP dynamics in frequency domain using harmonic transfer functions [14, 21]. However, the assumption of smooth dynamics does not readily apply to many rhythmic behaviors that involve hybrid dynamic characteristics.

In order to fill these gaps, we propose a new formulation for hybrid rhythmic dynamic systems using discrete time harmonic transfer functions that enables us to perform inputoutput system ID in frequency domain. 


\section{Hybrid DyNAMiCAL SyStem Formulation With EXOGENOUS INPUT}

The section follows briefly summarizes the development of [10] and modifies it to include exogenous inputs.

The state space of a hybrid system is a union

$$
V=\bigcup_{\alpha \in \mathcal{I}} V_{\alpha}
$$

where $\mathcal{I}$ is a finite index set and each $V_{\alpha}$ is an open, connected subset of $\mathbb{R}^{n_{\alpha}}$. Each element of this union, i.e. $V_{\alpha}$, is called a chart The dimension of the charts can typically depend on $\alpha[5,6,20]$. A state of the overall hybrid dynamical system consists of an index $\alpha$ together with a point in the chart $V_{\alpha}$. We assume that a (smooth) continuous time dynamical system is defined on each chart. Since $V_{\alpha} \subset R^{n_{\alpha}}$ we can represent the smooth dynamical system on each chart using

$$
\dot{q}_{\alpha}=f_{\alpha}\left(q_{\alpha}, u_{\alpha}, t\right)
$$

where $\dot{q}_{\alpha} \in V_{\alpha}$ is the state of the vector-field associated with chart $\alpha$ and $u \in \mathbb{R}^{l_{\alpha}}$ represents the small external perturbations used for system identification. One should also note that, similar to the state vector $q_{\alpha}$, dimension of the exogenous input may depend on $\alpha$ for hybrid dynamical systems. Indeed, exogenous inputs may not available $\left(l_{\alpha}=\right.$ 0 ) for some charts. As a convenience of notation, and without loss of generality we assume that there exists a $\hat{u} \in \mathbb{R}^{l_{\max }}$ which can be freely controlled by the experimenter, and a set of coordinate dependent mappings, $g_{\alpha}: \mathbb{R}^{l_{\max }} \rightarrow \mathbb{R}^{l_{\alpha}}$, such that $l_{\max }=\max _{\alpha \in \mathcal{I}} l_{\alpha}$ and $u_{\alpha}=g_{\alpha}(\hat{u}), \forall \alpha$. These maps should be locally onto (so as not to throw away "useful" perturbation inputs), and without loss of generality, $g_{\alpha}(0)=0$. On charts for which $l_{\max }>l_{\alpha}$, the extra inputs are "thrown away" by the dynamics. Under these constraints (1) takes the form

$$
\dot{q}_{\alpha}=f_{\alpha}\left(q_{\alpha}, \hat{u}, t\right)
$$

We assume that for each $\alpha \in I$, there exists a real-valued, piecewise-smooth threshold function, $h_{\alpha}^{\beta}\left(q_{\alpha}, \hat{u}\right)$. Zero crossings of a threshold function is called an event which triggers the transition to a new chart indexed by $\beta$. We further assume that there are transition maps, $q_{\beta}=T_{\alpha}\left(q_{\alpha}, \hat{u}\right)$ that apply a transformation to the points at an event. The images of the transition maps are taken as initial conditions for the continuous-time trajectory inside the new chart. Conceptually, the evolution of the system is viewed as a sequence of trajectory segments where the endpoint of one segment is connected to the initial point of the next by a transition map.

Given an input $\hat{u}(t)$, an initial chart $\alpha_{0}$, and initial condition on that chart $q_{\alpha_{0}}\left(t_{0}\right)$, we define a trajectory on the interval $\left[t_{0}, t_{n}\right]$ as follows. Denote events $t_{1}, \ldots, t_{n-1}$, such that $t_{0}<t_{1}<\cdots<t_{n}$. Each event time corresponds to a hybrid transition, giving rise to a sequence of discrete states $\alpha_{0}, \cdots, \alpha_{n-1}$ and smooth trajectories $q_{\alpha_{i}}(t) \in V_{\alpha_{i}}, t \in$ $\left[t_{i}, t_{i+1}\right]$, such that each $q_{\alpha_{i}}$ is a trajectory of the continuoustime dynamical system on $V_{\alpha_{i}}$ given in (2). Further, the

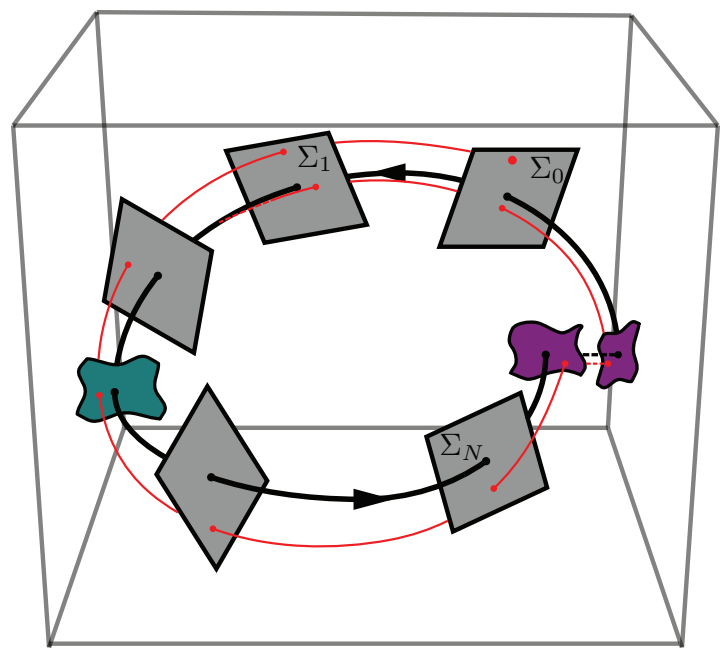

Fig. 1. Illustration of a stable rhythmic hybrid dynamical system with two charts, i.e. $\mathcal{I}=\{0,1\}$. For simplicity, each chart has the same dimension, and is a subset of $\mathbb{R}^{3}$. The limit cycle of the system (black) is discontinuous. The two-dimensional surface (green) illustrates a hybrid transition (patch) boundary in which the transition is continuous (no jump in continuous variables of the state space) but not necessarily differentiable. The pair of surfaces (purple) connected by dashed lines illustrate a hybrid transition boundary in which the transition is discontinuous. The two-dimensional cross-sections (grey) illustrate the $N$ Poincaré sections chosen by the experimenter. (These can be the hybrid boundaries, but in this example are chosen not to be.) The red curve represents a sample trajectory starting from an initial condition located at $\Sigma_{0}$. As illustrated in the figure, in the absence of external inputs trajectory converges to the limit cycle.

initial condition for each trajectory is given by $q_{\alpha_{i}}$, and for $i=1, \ldots, n-1$, these are calculated via the transition map: $q_{\alpha_{i}}\left(t_{i}\right)=T_{\alpha_{i-1}}\left(q_{\alpha_{i-1}}\left(t_{i}\right), \hat{u}\left(t_{i}\right)\right)$.

In the absence of external inputs i.e. $\hat{u}(t)=0 \forall t$, we assume the existence of an isolated, hybrid period orbit, or limit cycle, $\gamma(t) \in V$, that satisfies

$$
\begin{aligned}
& \lim _{\hat{t} \rightarrow t^{-}}[\gamma(\hat{t})-\gamma(\hat{t}+T)]=0, \\
& \lim _{\hat{t} \rightarrow t^{+}}[\gamma(\hat{t})-\gamma(\hat{t}+T)]=0 .
\end{aligned}
$$

Similar to the previous work on rhythmic hybrid dynamical systems [6], we limit our attention to hybrid systems undergoing a finite number of isolated hybrid transitions near the limit cycle. We also assume that in a local neighborhood of the limit cycle that the number and order of hybrid transitions is fixed (although the transition times can and typically will vary) and both the threshold functions and transition maps associated with these transitions are locally smooth.

\section{Mapping Between Poincaré Sections}

A Poincaré section, $\Sigma_{i} \subset V$, is an embedded submanifold that intersects the periodic orbit at exactly one point [6]:

$$
\begin{aligned}
\left\{\left(\epsilon_{i}, \alpha_{i}\right)\right\} & =\gamma \cap \Sigma_{i}, \alpha_{i} \in \mathcal{I}, \epsilon_{i} \in V_{\alpha_{i}}, \\
\operatorname{dim}\left(\Sigma_{i}\right) & =\operatorname{dim}\left(V_{\alpha_{i}}\right)-1
\end{aligned}
$$

where $\left(\epsilon_{i}, \alpha_{i}\right)$ is called the fixed point of $\Sigma_{i}$. The flow should be transverse to $\Sigma_{i}$ in a neighborhood of $\epsilon_{i}$ for all sufficiently small inputs. Let $W_{i} \subset \Sigma_{i}$ be an open subset containing $\epsilon_{i}$. 
Let $\Sigma_{j}$ be another Poincaré section with associated fixed point $\left(\epsilon_{j}, \alpha_{j}\right)$. We define a mapping, $P_{i \rightarrow j}: W_{i} \times R^{l_{\max }} \rightarrow$ $\Sigma_{j}$ by assuming $\hat{u}=u_{i}$ is constant (i.e. a "zero-order hold") and tracing the hybrid trajectories from $q_{i} \in W_{i}$ forward in time until it intersects $\Sigma_{j}$ at $q_{j}$ :

$$
q_{j}=P_{i \rightarrow j}\left(q_{i}, u_{i}\right) .
$$

We assume that $P_{i \rightarrow j}$ is a well-defined and smooth map around $\left(\epsilon_{i}, 0\right)$. If $\Sigma_{i}=\Sigma_{j}$ and $u_{i}=0$ this maps becomes the more familiar Poincaré return map. It is natural to assume that our measurements of states $q_{i}$ are indirect and though a smooth mapping:

$$
z_{i}=h_{i}\left(q_{i}, u_{i}\right)
$$

Linearizing state and output mappings in (3) and (4) around $\left(q_{i}, q_{j}, u_{i}\right)=\left(\epsilon_{i}, \epsilon_{j}, 0\right)$ yields

$$
\begin{aligned}
x_{j} & =A_{i} x_{i}+B_{i} u_{i}, \\
y_{i} & =C_{i} x_{i}+D_{i} u_{i} .
\end{aligned}
$$

It is worth noting that $A_{i}$ need not be square, depending on the dimensions of $\Sigma_{i}$ and $\Sigma_{j}$. Now assume that we have $N$ distinct isolated sequential (in the order they are punctuated by the limit cycle) Poincaré sections indexed by $\{0,1, \cdots, N-1\}$; see Fig. 1 . We formulate the linearized mapping between consecutive sections using

$$
\begin{aligned}
x[n+1] & =A[n] x[n]+B[n] u[n], \\
y[n] & =C[n] x[n]+D[n] u[n],
\end{aligned}
$$

where $A[n]=A[n+N]$ (and likewise for $B, C, D$ ).

The linearized mappings between successive Poincaré sections forms a linear discrete time periodic (LDTP) dynamical system which facilitates input-output modeling. Using (6) we can compute the linearized Poincaré return map at any section. Let $i \in\{0, \cdots, N-1\}$ be the section that we are interested. Then,

$$
D P_{i \rightarrow i}=\prod_{k=i+N-1}^{i} A[k]=A[i+N-1] \cdots A[i]
$$

yields the linearized Poincaré return map at that section. It is obvious that for hybrid systems in which the dimension of charts $\left(\operatorname{dim}\left(V_{\alpha}\right)\right)$ changes with $\alpha$, the linearized Poincaré return map in (7) is always rank-deficient for some $i \in$ $\{0, \cdots, N-1\}$ [20]. Even with rank deficiencies and the dimension of $x[n]$ is time varying, the liner time varying dynamics in (6) is suitable for deriving harmonic transfer functions using impulse response representation.

\section{HARMONiC TRANSFER Functions (HTF) FOR Linear Discrete Time Periodic Systems (LDTP)}

The computations above in Section III demonstrate that hybrid dynamical systems, operating near a limit cycle, can be approximated using a LDTP system. However, a transfer function representation may be more amenable to input-output system identification. In this section we will reformulate the derivations in [14] to suit LDTP systems.
Any (causal) LDTP systems such as in (6) can be represented using time-periodic impulse response

$$
y[n]=\sum_{k=0}^{n} H[n, k] u[k]
$$

where $H[n, k]=H[n-N, k-N]$. For the sake of clarity of derivations, throughout the paper we assume that $N$ is even (easily relaxed). Let $k=n-r$ then $H[n, k] \mapsto H[n, n-r]$ which is periodic in $n$ for any fixed $r$ thus can be expressed as a Fourier series:

$$
H[n, n-r]=\sum_{m=-\frac{N}{2}}^{\frac{N}{2}-1} H_{m}[r] e^{i \frac{2 \pi m}{N} n}
$$

where

$$
H_{m}[r]=\frac{1}{N} \sum_{n=\frac{-N}{2}}^{\frac{N}{2}-1} H[n, n-r] e^{-i \frac{2 \pi m}{N} n}
$$

Plugging $r=n-k$ gives

$$
H[n, k]=\sum_{m=-\frac{N}{2}}^{\frac{N}{2}-1} H_{m}[n-k] e^{i \frac{2 \pi m}{N} n} .
$$

Combining (8) and (11), $y[n]$ can be written as

$$
y[n]=\sum_{m=\frac{-N}{2}}^{\frac{N}{2}-1}\left(H_{m}[n] e^{i \frac{2 \pi m}{N} n} * u[n] e^{i \frac{2 \pi m}{N} n}\right) .
$$

Taking the Z-transform of $y[n]$,

$$
Y(z)=\sum_{m=-\frac{N}{2}}^{\frac{N}{2}-1} H_{m}\left(z e^{-i \frac{2 \pi m}{N}}\right) U\left(z e^{-i \frac{2 \pi m}{N}}\right)
$$

where

$$
H_{m}(z)=\mathcal{Z}\left\{H_{m}[n]\right\} .
$$

We can obtain the frequency response version of the HTF equation in (12) using the mapping $z \mapsto e^{i w}$ :

$$
Y(\omega)=\sum_{m=\frac{-N}{2}}^{\frac{N}{2}-1} H_{m}\left(\omega-\frac{2 \pi m}{N}\right) U\left(\omega-\frac{2 \pi m}{N}\right) .
$$

$H_{m}(z)$ (or $H_{m}(w)$ ) is the $m^{\text {th }}$ harmonic of the HTF structure that defines the coupling between the output at frequency $w\left(\right.$ or $\left.w+\frac{2 \pi m}{N}\right)$ and the input at frequency $w-\frac{2 \pi m}{N}$ (or $\left.w\right)$.

\section{IDENTIFICATION OF HTF OF LDTP SYSTEMS}

\section{A. Identification via Single Cosine Inputs}

In this section we show how harmonic transfer functions can be estimated using single cosine inputs and the limitations of this approach. Let the input be a (real) phase shifted cosine signal $u[n]=\frac{a}{\pi} \cos (\bar{\omega} n+\phi), \bar{w} \in[0, \pi)$. If we use the 
HTF structure in (14), the response to $u[n]$ can be computed in frequency domain as

$$
\begin{aligned}
Y(\omega) & =\sum_{m=\frac{-N}{2}}^{\frac{N}{2}-1} H_{m}\left(\omega-\frac{2 \pi m}{N}\right) a e^{i \phi} \delta\left(\omega-\frac{2 \pi m}{N}-\bar{\omega}\right) \\
& +\sum_{m=\frac{-N}{2}}^{\frac{N}{2}-1} H_{m}\left(\omega-\frac{2 \pi m}{N}\right) a e^{-i \phi} \delta\left(\omega-\frac{2 \pi m}{N}+\bar{\omega}\right)
\end{aligned}
$$

Let us analyze the steady-state frequency response for $\omega=$ $\bar{\omega}+\frac{2 \pi l}{N}$ where $l \in\{-N / 2, \ldots, N / 2\}$ :

$$
\begin{aligned}
Y\left(\bar{\omega}+\frac{2 \pi l}{N}\right) & =H_{l}(\bar{\omega}) a e^{i \phi} \\
& +\operatorname{sgn}\left(m^{*}\right) H_{l-m^{*}}\left(\bar{\omega}+\frac{2 \pi m^{*}}{N}\right) a e^{-i \phi}
\end{aligned}
$$

where

$$
m^{*}=\left\{\begin{aligned}
\frac{\bar{\omega} N}{\pi}, & \text { if } \frac{\bar{\omega} N}{\pi} \in \mathbb{Z}^{+} \\
0, & \text { otherwise }
\end{aligned}\right.
$$

If $m^{*} \neq 0$ then, there exist two unknowns, thus it is not possible to identify neither $H_{l}(\bar{\omega})$ nor $H_{l-m^{*}}\left(\bar{\omega}+\frac{2 \pi m^{*}}{N}\right)$ using a single cosine input. However if $m^{*}=0$ (or equivalently $\frac{\bar{\omega} N}{\pi} \notin \mathbb{Z}^{+}$), then we can identify $H_{l}(\bar{\omega})$ via

$$
H_{l}(\bar{\omega})=\frac{Y\left(\bar{\omega}+\frac{2 \pi l}{N}\right)}{a e^{i \phi}} .
$$

In conclusion, in order to identify the elements of HTF using single cosine inputs (for each frequency), the input frequency must not be an integer multiple of half of the socalled "pumping frequency" $\left(\omega_{p}=\frac{2 \pi}{N}\right)$, i.e., $\frac{\bar{\omega} N}{\pi} \notin \mathbb{Z}^{+}$.

Note that it is possible to identify the elements of the HTF at these harmonics by simply exciting the system using two cosine inputs (same frequency, different phase) [11]. However, if we assume the HTFs are smooth and the resolution of the DFT frequencies are high enough, the HTFs at these frequencies can be interpolated in order not to increase the number of experiments.

\section{B. Identification via Sums of Cosine Inputs}

In this section our goal is to determine the rules such that elements of the HTF can be estimated uniquely (assuming no noise) from the experiments where the input stimulus is the sum of cosine inputs. Let $\left\{\bar{\omega}_{1}, \cdots, \bar{\omega}_{K}\right\},\left\{\phi_{1}, \cdots, \phi_{K}\right\}$, and $\left\{\frac{a_{1}}{\pi}, \cdots, \frac{a_{K}}{\pi}\right\}$ be the set of input frequencies, phase shifts, and magnitudes respectively. The input signal for the experiment can be constructed as

$$
u[n]=\sum_{k=1}^{K} \frac{a_{k}}{\pi} \cos \left(\bar{\omega}_{k} n+\phi_{k}\right) .
$$

Since single sine experiment is a special case of a sum of sine experiment, the obvious first rule for the identification is

$$
\frac{\bar{\omega}_{k} N}{\pi} \notin \mathbb{Z}^{+} \forall k
$$

For the sake of clarity let's assume that $K=2$. Then frequency response of the system to the input (15) at the frequency $\omega=\bar{\omega}_{1}$ takes the form

$$
\begin{aligned}
Y\left(\bar{\omega}_{1}\right) & =H_{0}\left(\bar{\omega}_{1}\right) a_{1} e_{1}^{i \phi} \\
& +\operatorname{sgn}\left(m_{1}^{*}\right) H_{m_{1}^{*}}\left(\bar{\omega}_{1}-\frac{2 \pi m_{1}^{*}}{N}\right) a_{2} e^{i \phi_{2}} \\
& +\operatorname{sgn}\left(m_{2}^{*}\right) H_{m_{2}^{*}}\left(\bar{\omega}_{1}-\frac{2 \pi m_{2}^{*}}{N}\right) a_{2} e^{-i \phi_{2}}
\end{aligned}
$$

where

$$
\begin{aligned}
& m_{1}^{*}=\left\{\begin{aligned}
\frac{\left(\bar{\omega}_{1}-\bar{\omega}_{2}\right) N}{2 \pi}, & \text { if } \frac{\left|\bar{\omega}_{1}-\bar{\omega}_{2}\right| N}{2 \pi} \in \mathbb{Z}^{+} \\
0, & \text { otherwise }
\end{aligned}\right. \\
& m_{2}^{*}=\left\{\begin{aligned}
\frac{\left(\bar{\omega}_{1}+\bar{\omega}_{2}\right) N}{2 \pi}, & \text { if } \frac{\left(\bar{\omega}_{1}+\bar{\omega}_{2}\right) N}{2 \pi} \in \mathbb{Z}^{+} \\
0, & \text { otherwise }
\end{aligned}\right.
\end{aligned}
$$

Similar structure is obtained if we extend to arbitrary $K$ and observe the response at $\omega=\bar{\omega}_{k}+\frac{2 \pi l}{N}$. From this result, we observe that in order to identify the elements of the HTF uniquely, in addition to the constraint on each individual frequency in (16), we also require that the difference and the sum of any two frequencies in the input stimulus must not be an integer multiple of the pumping frequency. Under these rules elements of the HTF structure can be estimated using

$$
H_{l}\left(\bar{\omega}_{k}\right)=\frac{Y\left(\bar{\omega}_{k}+\frac{2 \pi l}{N}\right)}{a_{k} e^{i \phi_{k}}} .
$$

Under these rules our system identification procedure using sum-of-sine inputs is as follows:

- Define the frequency band of interest, $\bar{\omega}_{k} \in\left(0, \omega_{\max }\right]$ and compute the resolution, $\omega_{\text {res }}$, of the DFT frequencies based on the length of the input-output data.

- Construct a global set of frequencies, $\Omega=$ $\left\{\omega_{r e s}, 2 \omega_{r e s}, 3 \omega_{r e s}, \cdots \omega_{\max }\right\}$.

- Remove the frequencies that are the integer multiples of half of the pumping frequency, i.e. $\bar{\Omega}=\Omega-$ $\left\{\frac{\pi}{N}, \frac{2 \pi}{N}, \frac{3 \pi}{N}, \cdots\right\}$.

- Partition $\Omega$ in to $S$ sub sets, $\bar{\Omega}=\Omega_{1} \cup \Omega_{2} \cdots \cup \Omega_{S}$, such that for each $\Omega_{s}$ following condition is satisfied:

$$
\begin{aligned}
\frac{\left|\bar{\omega}_{i}-\bar{\omega}_{j}\right| N}{2 \pi} \notin \mathbb{Z}^{+} \& \frac{\left(\bar{\omega}_{i}+\bar{\omega}_{j}\right) N}{2 \pi} \notin \mathbb{Z}^{+} \\
\forall \bar{\omega}_{i}, \bar{\omega}_{j} \in \Omega_{S}
\end{aligned}
$$

- For each subset construct an input stimulus

$$
u_{s}[n]=\sum_{\bar{\omega}_{k} \in \Omega_{s}} \frac{a_{k}}{\pi} \cos \left(\bar{\omega}_{k}+\phi_{k}\right),
$$

and perform the experiment to compute the elements of HTF.

\section{RESULTS AND DISCUSSION}

\section{A. Example Model System}

We apply our system identification framework to the vertical hopper model illustrated in Fig. 2. We adopted the model from [5] and added an exogenous input $u$ acting on the upper body mass $M$ to be used for system identification. 


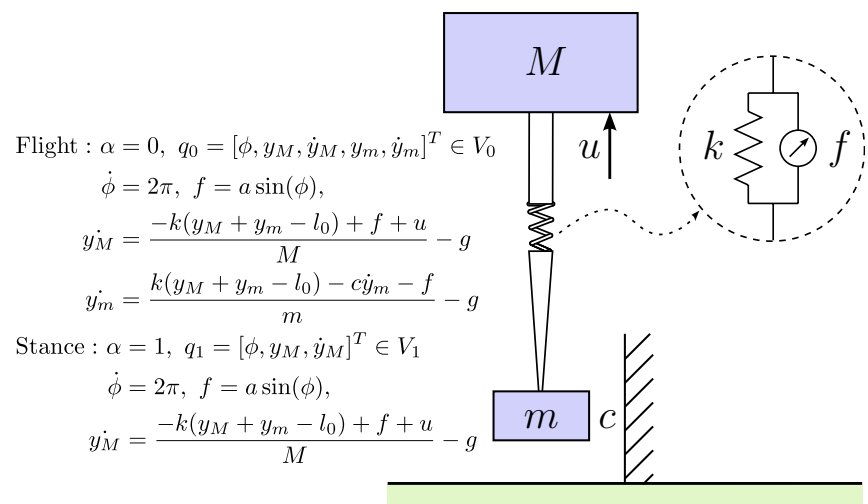

Fig. 2. Dynamics and schematic of the hopper model

The "clock-driven" hopper alternates between flight $(\alpha=0)$ and stance $(\alpha=1)$ charts during hopping. Flight and stance dynamics of the hopper are embedded in Fig. 2.

The touchdown event defines the transition from flight to stance chart. The zero crossing of the threshold function, $h_{o}\left(q_{0}\right)=y_{m}$, triggers the transition and the states of the new chart is defined by the transformation $q_{0} \mapsto T_{0}\left(q_{0}\right)$ where $T_{0}\left(q_{0}\right)=\left[\begin{array}{lll}I_{3 \times 3} & 0_{3 \times 2}\end{array}\right] q_{0}$. The liftoff event defines the transition from stance to flight, which is triggered by the zero crossing of the threshold function $h_{1}\left(q_{1}\right)=k\left(l_{0}-\right.$ $\left.y_{M}\right)+f+m g$. The initial state of the new flight chart is determined by the transformation $q_{1} \mapsto T_{1}\left(q_{1}\right)$ where $T_{1}\left(q_{1}\right)=\left[\begin{array}{lll}I_{3 \times 3} & 0_{3 \times 2}\end{array}\right]^{T} q_{1}$. We simulated the hybrid hopper model in MATLAB (Mathworks, Inc.), using a custom hybrid dynamical simulation toolkit with the sampe parameters used in [5]. We verified with our simulation toolkit that this set of parameters (when external input $u=0$ ) produces a stable limit cycle. The non-zero eigenvalues of the Poincaré maps of the system are $\lambda_{1,2}=0.25 \pm 0.7 i$, computed via the numerical Jacobian of the return map.

\section{B. Phase Coordinates and Set of Poincaré Sections}

As detailed in Sec. III our system identification method relies on mappings between selected discrete phase coordinates (i.e. set of Poincaré Sections). We expect that the structure of HTF depends on the selected phase coordinates.

In this paper, we utilized two "causal" phase definitions to select Poincaré sections. Existence of exogenous input and its dependence on phase coordinates makes the straight forward implementation of non-causal methods [16] impossible. The hybrid system we are analyzing is an asymptotically stable clock-driven model, thus one natural way of selecting Poincaré sections is using the isochrons [9] which are directly given by the phase of the clock. We select $N$ equally spaced Poincare sections within $\phi \in[0,2 \pi)$. The phase of the $i^{t h}$ Poincaré section corresponds to $\phi_{i}=\frac{2 \pi}{N} i$.

However, the majority of rhythmic hybrid system models are not driven by an open-loop clock thus there is no straightforward way of accessing the isochrons, especially using a causal method. The main advantage of our method is that it works with any set of well defined Poincaré sections.
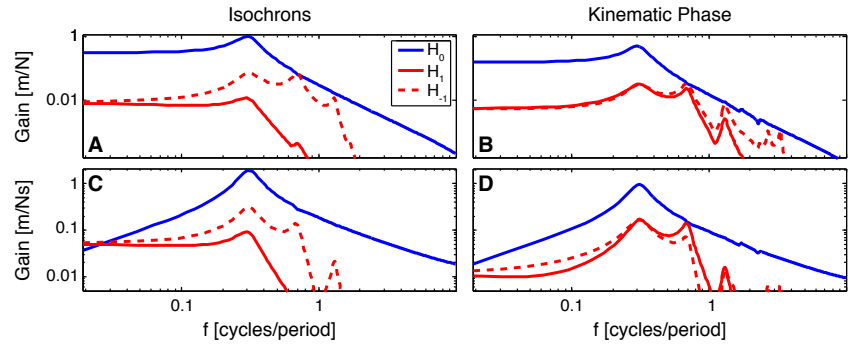

Fig. 3. Non-parametric estimates of $\left|H_{0}(z)\right|,\left|H_{1}(z)\right|$ and $\left|H_{-1}(z)\right|$. Magnitude plots in the top row (A and B) represens the HTFs between the input and $\delta y_{M}$, where as bottom row (C and D) belongs to the HTFS between the input and $\delta \dot{y}_{M}$. First (A and $\left.\mathrm{B}\right)$ and second column (C and D) represents the HTFs where the phase coordinates are selected using isochrons, and kinematic phase respectively.

In addition to isochrons, we use a definition similar to the kinematic phase used in [15]. Using the states $y_{M}, \dot{y}_{M}$, we define a phase variable:

$$
\begin{aligned}
& r_{p}=\frac{y_{M}-o_{p}}{s_{p}}, r_{v}=\frac{\dot{y}_{M}-o_{v}}{s_{v}} \\
& \hat{\phi}=\not r_{p}+i r_{v}, \hat{\phi} \in S^{1}
\end{aligned}
$$

where $\left(o_{p}, o_{v}\right)$ and $\left(s_{p}, s_{v}\right)$ simply shifts the origin and scales the coordinates, respectively. Based on this definition of phase, we select equally spaced $N$ Poincare sections within $\hat{\phi} \in[0,2 \pi)$. In our simulations, for both isochrons and kinematic phase, we choose $N=32$ and align the $\phi=0$ and $\hat{\phi}=0$ events on the limit cycle.

\section{Simulation Results}

1) Non-parametric Harmonic Transfer Functions: In this section we present the estimated non-parametric harmonic transfer functions based on two different phase coordinates: isochrons and kinematic phase with $\left(o_{p}, o_{v}, s_{p}, s_{v}\right)=$ $(1.9,0,0.3,1.9)$.

We identify the HTF blocks from $u[n]$ to $y[n]$, where $y$ is either taken as the deviation of the height from the fixed point, namely $\delta y_{M}$, or the deviation of the vertical velocity from the fixed point, $\delta \dot{y}_{M}$. In order to compute the limitcycles, we run the hybrid simulation script in the absence of any external input for $60 \mathrm{~s}$ and use the last cycle of this simulation as the baseline for the limit cycle. We use the method proposed in V-B and excite the system with inputs that are sums of cosine signals. The length of the each sums of cosines experiment is 84 cycles, however we only use the last 64 cycles in order not to capture any transient effects on the frequency response data. 64 cycles of data with $N=32$ provides a frequency resolution of $f_{\text {res }}=\frac{1}{32}$ (or $\omega_{\text {res }}=\frac{\pi}{16} \mathrm{rad} / s$ ). $f$ is the normalized frequency with units of cycles per fundamental period. We compute the HTFs in the frequency band $f \in(0,10)$. As explained in Sec.V-A we remove the frequencies that are the prime multiple of the $f=0.5$ (or $\omega=\frac{\pi}{32}$ ) and we were left with 620 frequencies for which we need to identify the HTF components. We divide these 620 frequencies into 80 subsets randomly following the rules derived in Sec. V-B. For each subset we construct an input signal, perform the experiment, 


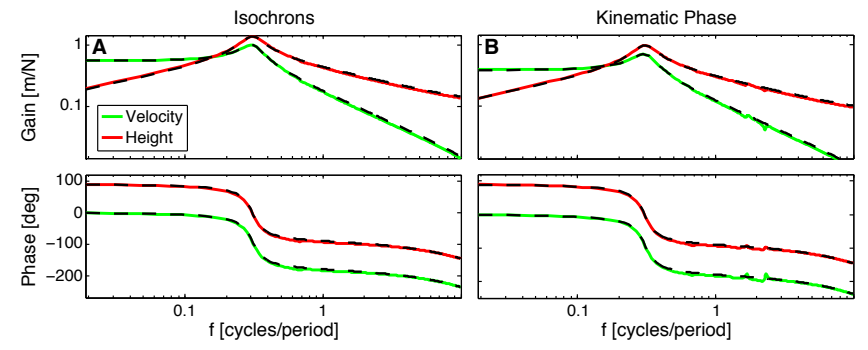

Fig. 4. Non-parametric and parametric estimates of $\left|H_{0}(z)\right|,\left|H_{1}(z)\right|$ and $\left|H_{-1}(z)\right|$.Figures in the top row indicates the magnitude plots, where as the ones in the bottom row indicates the phase plots. Green and red curves represents the magnitude and phase plots for the HTFS from input to $\delta y_{M}$ and from input to $\delta \dot{y}_{M}$ respectively. Black curves the magnitude and phase plots of the estimated parametric transfer functions. First (A) and second column (B) represents the HTFs where the phase coordinates are selected using isochrons and kinematic phase respectively.

and compute the HTF components associated with those frequencies.

Fig. 3 illustrates the magnitude plots of the empirical HTFs. For all phase coordinates we found that the magnitude of the components, $H_{i}$, for $|i| \geq 2$ were negligible compared to $\left|H_{0}(z)\right|,\left|H_{1}(z)\right| \&\left|H_{-1}(z)\right|$ thus we only illustrate those three responses. This is somewhat surprising, because even for a hybrid system for which the dimension of charts are not constant three harmonics can accurately describe the dynamics around the limit cycle.

2) Phase Coordinates Affect Zeros, not Poles: If we compare the HTFs from different phase coordinates, we can see that the structure of the HTF is indeed affected by the choice of Poincaré sections. In the context of $H_{0}(z)$ the gain is doubled if we use isochrons instead of the kinematic phase definition (note the vertical shift in Fig. 3 in the blue curve). The phase coordinates affects the harmonics $H_{1}(z)$ and $H_{-1}(z)$ structurally (red and dashed red curves). However, it seems that the "resonant" frequencies (peaks of the magnitude plots) are located at approximately same locations for all phase coordinates. We suspect that this is because choice of Poincaré sections do not move the pole dynamics of the HTF but instead changes the zeros.

3) Estimation of Poincaré Return Map Eigenvalues Through HTF: In this section, we present an approximate parametric estimation approach for the non-zero eigenvalues of the Poincaré return map through parametric identification of the fundamental transfer function, i.e. $H_{0}(z)$. If we observe the bode plots of $H_{0}(z)$ illustrated in Fig. 4, we see that all of them approximately look like transfer functions with two poles, so we assumed a second-order transfer function with one zero and two poles.

From the results we see that the parametric transfer functions fit the frequency response data very well except some higher order "bumps" around $f=2 \mathrm{~Hz}$, i.e. twice the system pumping frequency. Since we have a parametric estimation of $H_{0}(z)$ we can now recover the eigenvalues of the Poincaré return map. Let $p$ be a pole of the HTF, then the eigenvalues of the Poincaré return map can be computed simply $\lambda=p^{N}$. All of the six parametric transfer functions were correctly estimated the true eigenvalues of the system $\lambda_{1,2}=0.25 \pm 0.70 i$ (with numerical errors at the third significant digit).

\section{ACKNOWLEDGMENTS}

This material is based on work supported by the National Science Foundation Grants 0845749 and 1230493 (to N. J. Cowan).

\section{REFERENCES}

[1] A. D. Ames, R. D. Gregg, E. D. Wendel, and S. Sastry. On the geometric reduction of controlled three-dimensional bipedal robotic walkers. In Lagrangian and Hamiltonian Methods for Nonlinear Control 2006, pages 183-196. Springer, 2007.

[2] M. M. Ankarali, H. T. Şen, A. De, A. M. Okamura, and N. J. Cowan. Haptic feedback enhances rhythmic motor control by reducing variability, not improving convergence rate. J Neurophysiol, 111(6):12861299, 2014.

[3] R. Blickhan and R. J. Full. Similarity in multilegged locomotion: Bouncing like a monopode. J Comp Physiol A, 173(5):509-517, 1993.

[4] M. Buehler, D. E. Koditschek, and P. J. Kindlmann. Planning and control of robotic juggling and catching tasks. Int J Robot Res, 13(2):101-118, 1994.

[5] S. Burden, S. Revzen, and S. S. Sastry. Dimension reduction near periodic orbits of hybrid systems. In Decision and Control and European Control Conference (CDC-ECC), 2011 50th IEEE Conference on, pages 6116-6121. IEEE, 2011.

[6] S. A. Burden, S. Revzen, and S. S. Sastry. Model reduction near periodic orbits of hybrid dynamical systems. arXiv preprint arXiv:1308.4158, 2013.

[7] S. G. Carver, N. J. Cowan, and J. M. Guckenheimer. Lateral stability of the spring-mass hopper suggests a two step control strategy for running. Chaos, 19(2), 2009.

[8] N. J. Cowan, M. M. Ankarali, J. P. Dyhr, M. S. Madhav, E. Roth, S. Sefati, S. Sponberg, S. A. Stamper, E. S. Fortune, and T. L. Daniel. Feedback control as a framework for understanding tradeoffs in biology. Integr Comp Biol, 54(2):223-237, June 2014.

[9] J. M. Guckenheimer. Isochrons and phaseless sets. J Math Biol, 1:259-273, 1975.

[10] P. J. Holmes, R. J. Full, D. E. Koditschek, and J. Guckenheimer. The dynamics of legged locomotion: Models, analyses, and challenges. SIAM Rev, 48(2):207-304, 2006.

[11] S. Hwang. Frequency domain system identification of helicopter rotor dynamics incorporating models with time periodic coefficients. $\mathrm{PhD}$ thesis, University of Maryland College Park, 1997.

[12] T. Kiemel, D. Logan, and J. J. Jeka. Using perturbations to probe the neural control of rhythmic movements, 2014. In preperation.

[13] D. E. Koditschek, R. J. Full, and M. Buehler. Mechanical aspects of legged locomotion control. Arthropod Structure \& Development, 33:251-272, 2004.

[14] E. Möllerstedt. Dynamic analysis of harmonics in electrical systems. $\mathrm{PhD}$ thesis, Department of Automatic Control, Lund Institute of Technology, 2000.

[15] S. Revzen. Neuromechanical Control Architectures of Arthropod Locomotion. PhD thesis, University of California, Berkeley, 2009.

[16] S. Revzen and J. M. Guckenheimer. Estimating the phase of synchronized oscillators. Phys Rev E, 78(5 Pt 1):051907-051918, 2008.

[17] U. Saranli, M. Buehler, and D. E. Koditschek. RHex: A simple and highly mobile hexapod robot. Int J Robot Res, 20(7):616-631, 2001.

[18] T. Söderström and P. Stoica. System Identification. Systems and Control Engineering. Prentice Hall International, 1989. Out of print.

[19] E. Tytell, P. Holmes, and A. Cohen. Spikes alone do not behavior make: why neuroscience needs biomechanics. Current opinion in neurobiology, 21(5):816-822, 2011.

[20] E. Wendel and A. D. Ames. Rank deficiency and superstability of hybrid systems. Nonlinear Analysis: Hybrid Systems, 6(2):787-805, 2012.

[21] N. M. Wereley. Analysis and Control of Linear Periodically Time Varying Systems. $\mathrm{PhD}$ thesis, Massachusetts Institute of Technology, 1990. 